\title{
Image Restoration using a Combinational Trivaritate Shrinkage Filter and PSO
}

\author{
Prabhjot Kaur \\ M-TECH Scholar (ECE) \\ RIEIT, RailMajra, Ropar
}

\begin{abstract}
Image restoration in digital image processing is a technique through which corrupted images are modified to increase the quality of the image. A lot of research work has been done in this contrast to make the image quality efficient enough. When the noise level in the image is low, it is easy to rectify the image using general filter but the procedure is not effective enough when the noise in the image is high. To rectify highly noised images we need combinational filter which divides the images into section and perform the threshold operation according to the sections divided. In this paper, we propose a unique methodology to perform image restoration using a combinational trivaraiate shrinkage filter \&PSO within the wavelet domain for $512 * 512$ pixel size image.
\end{abstract}

\section{Keywords}

Noise Removal, Trivariate Shrinkage Filter, PSO, Digital Image Processing

\section{INTRODUCTION}

Image restoration is basically improving the interpretability or perception of information in images for human viewers and providing 'better' input for other automated image processing techniques. The principal objective of image restoration is to modify attributes of an image to make it more suitable for a given task and a specific observer. During this process, one or more attributes of the image are modified. The choice of attributes and the way they are modified are specific to a given task. Moreover, observer-specific factors, such as the human visual system and the observer's experience [1], will introduce a great deal of subjectivity into the choice of image restoration methods. There exist many techniques that can enhance a digital image without spoiling it. The restoration methods can broadly be divided in to the following two categories:

1. Spatial Domain Methods

2. Frequency Domain Methods [3]

\subsection{Spatial Domain Method}

It is used for manipulating or changing an image representing an object in space to enhance the image for a given application.The techniques are based on direct manipulation of pixels in an image.It is Used for filtering basics, smoothing filters, sharpening filters, unsharp masking and laplacian.

\author{
Sukhwinder Kaur \\ Asst. Prof (ECE) \\ RIEIT, RailMajra Ropar
}

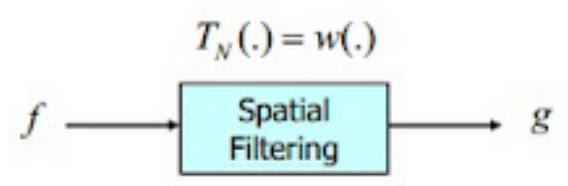

$$
\begin{gathered}
g(m, n)=\sum_{i=-a j=b}^{b} \sum^{b} w(i, j) f(m+i, n+j) \\
1 \leq m \leq M \\
1 \leq n \leq N
\end{gathered}
$$

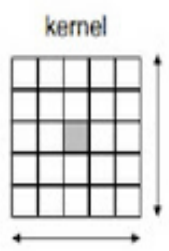

Fig 1: Kernal Operator/ Filter Mask

\subsection{Frequency Domain Method}

The frequency domain techniques are based on modifying the spectral transform of an image and to transform the image to its frequency representation. It computes inverse transform back to the spatial domain. It has high frequencies correspond to pixel values that change rapidly across the image (e.g. text, texture, leaves, etc.) and strong low frequency components correspond to large scale features in the image (e.g. a single, homogenous object that dominates the image).

The technique used in frequency domain is Fourier transform. These are the function that are not periodic but with finite area under the curve can be expressed as he integral of sines and/ or sines multiplied by a weight function.

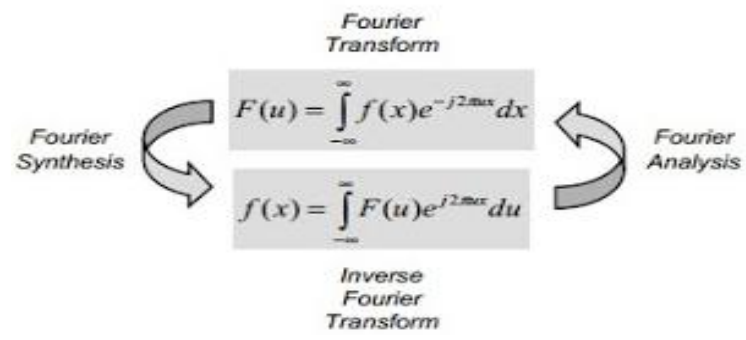

The best possible way to derive the noise level is the histogram pattern of the image. In this pattern we get the peaks of the noisy image as well as the original image and it clearly identifies that what is the level of the noise in the image. The following figure represent two images and their histogram representation. 


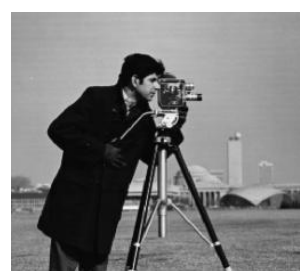

(a)Noise Free Image

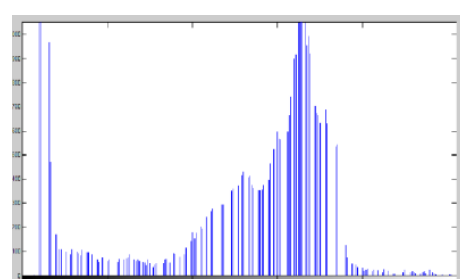

(b) Noise Free Histogram

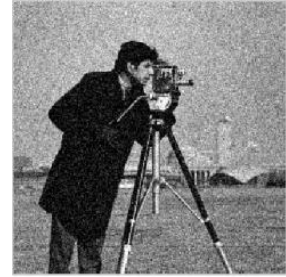

(c) Image with Noise

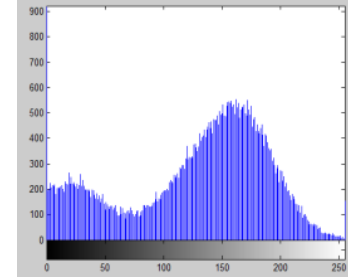

(d) Noisy Histogram

Fig 2: Difference in the noisy and the Noise free image.

From the above shown figures, it is quite obvious that the histogram can clearly show the difference in the Noisy and Noise free image.

This method is effective when the noise level is low but for highly noised image normal histogram method won't be sufficient enough to work on. Hence for the same we need a combinational filter. Our proposed work has following set of filters working.

In the previous approach bilateral filter is used which divides the entire segment into two parts and proceed in binary forms. The bilateral filter is also helpful in edge preserving. The Dyadic Wavelet transformation is used to convert the gray scale image into the wavelet transformation. This method is only suitable for wavelet transformations. Bayes filters are used for large size images. They work with a fixed pattern and check if the adjacent nodes are bayed then it is considered that entire pixel part would be bayed that means there would be no noise present in that. The multi scale product rule is a unique rule which adjusts the threshold of the sections according to the size of the section and the size of the image. Varying threshold is a good idea because noise in an image is never uniform and keeping same threshold for each and every section may harm the image lot.

\section{COMBINATIONAL FILTER \\ 2.1Trivariate Shrinkage Filter}

A trivariate filter divides the entire image in three segments [2].It generate threshold according to the soft threshold method for each sub block and perform de noising for each sub block separately .Finally the image in three segments is reconstructed.
Output Image $=\sum$ Image_PartCounter

where $i$ is the number of segmented noise free image

\subsection{PSO}

Particle Swarm Optimization is not exactly a filter. It is a methodology which can be used as a filter for the processing. PSO treats the image as a vector [3] and it generates weights for the input image and uses its fitness function [7] to optimize the result finally. A candidate vector $\mathrm{CN}=\{\mathrm{c} 1$, $\mathrm{c} 2, \ldots . \mathrm{cN}\}$ whose elements are the pixel locations from the search region $\mathrm{S}$ but not the member of L(local vector of Noisy Pixels),[5]

Let $\mathrm{PN}=\{\mathrm{p} 1, \mathrm{p} 2, \ldots, \mathrm{pN}\}$ be the vector of non noisy pixels corresponding to the elements in the vector $\mathrm{CN}$. The vector $\mathrm{PN}$ has been reformulated to $\mathrm{PMN}=\{\mathrm{P} 1 \mathrm{~N}, \mathrm{P} 2 \mathrm{~N}, \mathrm{P} 3 \mathrm{~N}\}$ where $\mathrm{P} 1 \mathrm{~N}$ is the number of non noisy pixels in $3{ }^{\prime} 3$ window, $\mathrm{P} 2 \mathrm{~N}$ is the number of non noisy pixels in $5^{\prime} 5$ window excluding P1N and $\mathrm{P} 3 \mathrm{~N}$ is the number of non noisy pixels in $7^{\prime} 7$ window excluding $\mathrm{P} 1 \mathrm{~N}$ and $\mathrm{P} 2 \mathrm{~N}$. Let $\mathrm{A} 1 \mathrm{~N}, \mathrm{~A} 2 \mathrm{~N}$ and $\mathrm{A} 3 \mathrm{~N}$ are the weight vectors generated by PSO during the course of algorithm corresponding to $\mathrm{P} 1 \mathrm{~N}, \mathrm{P} 2 \mathrm{~N}$ and $\mathrm{P} 3 \mathrm{~N}$ respectively such that $1 \mathrm{~N} 1 \mathrm{~A}=\mathrm{X}, 2 \mathrm{~N} 2 \mathrm{~A}=\mathrm{X}, 3 \mathrm{~N} 3 \mathrm{~A}=\mathrm{X}$ and $\Sigma \mathrm{X}=1$. The image is restored by applying the weights AMN.

\section{PROPOSED METHODOLOGY}

The trivariate shrinkage filter is one of the finest filter in the series of filters. Our proposed methodology have included the trivarate shrinkage filter along with PSO in such a manner that the best proposed solution for the image restoration still goes for best possible modifications. The flow diagram of our approach is as follows.

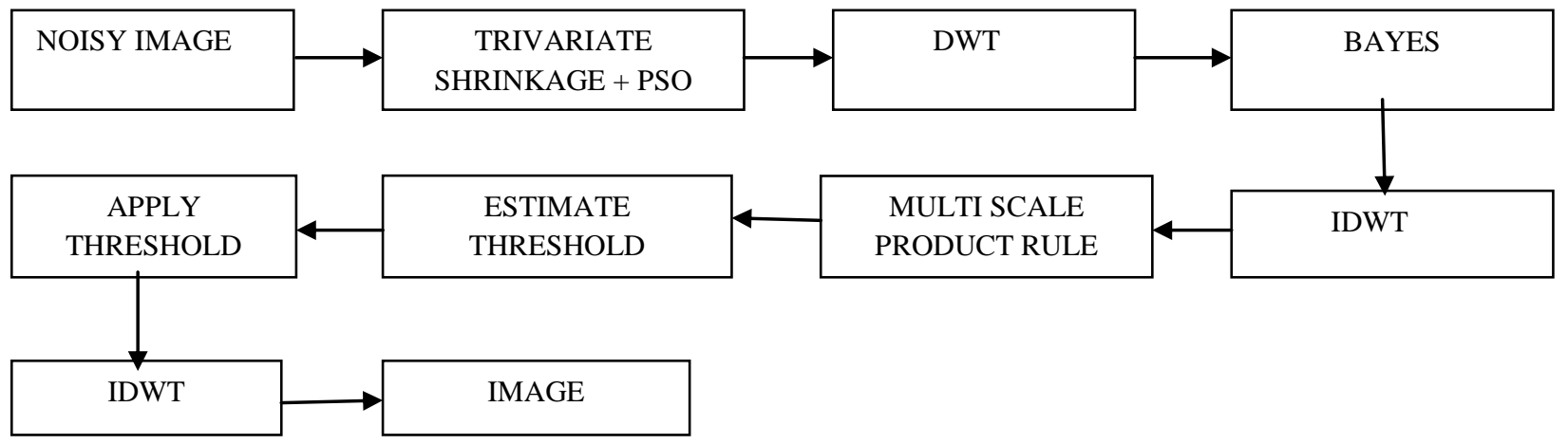

Fig.3: The flow structure of proposed methodology

In our proposed methodology we have worked with different images using the size of $512 * 512$.In this methodology we take different image of the same size and add noise in the image. We have also tested our algorithm by increasing the noise by 
$50 \%$ in it. Our proposed methodology follows following

\section{RESULTS AND CONCLUSION}

On the basis of the above algorithm we tested our results on Leena, Barbara, Boat and Peppers Image and the results are as follows. algorithm (hybrid ) to proceed.

\begin{tabular}{|c|c|c|c|c|c|c|c|c|c|c|}
\hline & \multicolumn{5}{|c|}{ PSNR(db) } & \multicolumn{5}{|c|}{ PSNR (db) } \\
\hline & \multicolumn{5}{|c|}{$\operatorname{Lena}(512 * 512)$} & \multicolumn{5}{|c|}{ Barbara $(512 * 512)$} \\
\hline Noise level & 10 & 20 & 30 & 40 & 50 & 10 & 20 & 30 & 40 & 50 \\
\hline $\begin{array}{l}\text { Previous } \\
\text { Approach }\end{array}$ & 33.91 & 31.0 & 29.7 & 28.4 & 27.0 & 31.7 & 29.4 & 26.9 & 24.5 & 24.1 \\
\hline $\begin{array}{l}\text { New } \\
\text { approach }\end{array}$ & 34.43 & 31.52 & 30.22 & 28.92 & 27.52 & 31.91 & 29.61 & 27.11 & 24.71 & 24.31 \\
\hline
\end{tabular}

\begin{tabular}{|l|l|l|l|l|l|l|l|l|l|l|l|}
\hline & \multicolumn{2}{l|}{ PSNR(db) } & \multicolumn{3}{l|}{ PSNR(db) } \\
\hline & \multicolumn{3}{|l|}{ Boat (512*512) } \\
\hline
\end{tabular}

The above table represents a comparative analysis of the result in which we can clearly see that the results through our proposed methodology is enhanced as compared to the previous results.

\section{FUTURE SCOPE}

The current methodology gives rise to a lot of future possibilities. We have used PSO algorithm which take the optimized result through its fitness function. There are other efficient algorithms also available which may optimize the current result also. In future instead of PSO, GA can be tried to optimize the result as it has better fitness function than PSO to optimize the result.

\section{REFERENCES}

[1] Raman Maini and Himanshu Aggarwal A Comprehensive Review of Image Restoration Techniques Journal Of Computing, Volume 2, Issue 3, March 2010, Issn 21519617 Https://Sites.Google.Com/Site/Journalofcomputing.

[2] Sumit A..Raokhande Image Denoising Using Trivariate Shrinkage Filter In Wavelet Domain'International Journal of Emerging Technology and Advanced Engineering Website: www.ijetae.com (ISSN 2250-2459, Volume 2, Issue 3, March 2012)
[3] S. Mohamed Mansoor Roomi, P.L. Muthu Karuppi A Particle Swarm Optimization Based Edge Preserving Impulse Noise Filter Journal of Computer Science 6 (9): 1014-1020, 2010 ISSN 1549-3636 @ 2010 Science Publications

[4] L. Sendur and I. W. Selesnick, "Bivariate shrinkage with local variance estimation," IEEE Signal Process. Lett, vol. 9, no. 12, pp. 438-441, Dec. 2002.

[5] J. Portilla, V. Strela, M. J. Wainwright, and E. P. Simoncelli, "Image denoising using scale mixtures of gaussians in the wavelet domain," IEEE Trans. Image Process., vol. 12, no. 11, pp. 1338-1351, Nov. 2003.

[6] M. Elad and M. Aharon, "Image denoising via learned dictionaries and sparse representation," IEEE Comput. Vis. Pattern Recognit., Jun. 2006.

[7] M. Elad and M. Aharon, "Image denoising via sparse and redundant representations over learned dictionaries," IEEE Trans. Image Process., vol. 15, no. 12, pp. 37363745, Dec. 2006.

[8] C. Tomasi and R. Manduchi, "Bilateral filtering for gray and color images," in Proc. Int. Conf. Computer Vision, 1998, pp. 839-846. 